\title{
Research of the Management Information System Comprehensive Assessment Model Based on BP Neural Network
}

\author{
Zhirong Liang ${ }^{1, a}$, Qi Xue ${ }^{1, b}$ and Dawang Cheng ${ }^{1, c}$ \\ ${ }^{1}$ Chinese people's Liberation Army Armored Forces Academy, Anhui, 233000, China

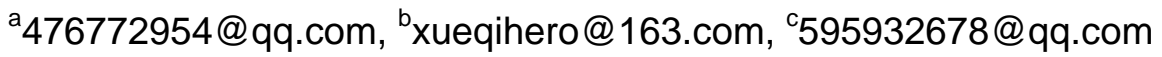

Keywords: Neural Networks, Management Information Systems, Comprehensive Assessment Model

\begin{abstract}
In this paper, on the basis of building management information systems (MIS) evaluation index system, we propose neural networks based integrated evaluation method for management information systems. It can simulate evaluation made by experts and avoid subjective mistakes. The results from the simulation are satisfied.
\end{abstract}

\section{Introduction}

Human society has entered the information society, in an increasingly competitive market, user demand tend to diversify, the degree of association between enterprises increasingly close today, the enterprise must act fast and sensitive. Therefore, enterprises manage information technology has become a corporate a very important condition for survival, but the development of information systems management is a systematic project, it takes a lot of manpower, material and financial resources and time, and its effect is often applied to build and put into use after a considerable period of time in order to reflect in the system out. thus prior scientific evaluation of the quality of information systems has very important significance. previous evaluations mostly by evaluators with a personal experience, which determine the existence of a subjective evaluation of arbitrary large, the accuracy is low and Unable to carry out a large-scale evaluation of defects, therefore, effective research, evaluation methods and evaluation index system of reliable management information system has become a hot research system development. Based on the evaluation system to establish a management information system based on the proposed a comprehensive index evaluation method based on neural network [1].

\section{Comprehensive Evaluation of Management Information Systems}

When evaluate the system, you first need to establish evaluation index system, and then to use appropriate methods for evaluation. Evaluation System management information system should include both information system developed to run, but also including direct users of information systems, That will also include the impact on the external environment.

Currently, the construction and operation of most of the information system maintenance is borne by the information center, so the system will build, operation, maintenance and management considered together their evaluation are:

Personnel information systems including configuration Leadership competent leadership information systems support the system construction, operation and maintenance support system running is an important factor in generating benefits. Advanced refers built systems in general is an advanced, and has a longer life cycle. Management Sciences of the so-called scientific management refers to whether a complete rules and regulations, duty system and logging systems, fire safety systems and systems. Maintainability of the system maintenance, expansion and modification It is absolutely regular. Resource utilization information system focused on a number of high-value-added equipment (hardware, software, and configuration of the system) as well as information and human resources, the degree of utilization of these resources should be a very important indicator. Development efficiency is construction speed refers to the information system. Investment in the system means it should establish a reasonable investment [2]. 
Safe and reliable management information system security and reliability generally refers to systems and information resources management information system is not natural and man-made threats and hazards and harmful factors. User index information systems from the viewpoint of the user information system referred to herein is direct users, including leaders and the middle and lower managers, a total of nine indicators: the importance of an information system for users in what position costs the economy information system users need to pay is reasonable timeliness of the information system user. And can be used to provide timely information is a matter of great interest to the user. Friendliness is the so-called user-friendly information system is very convenient, well-meaning man-machine interface. The accuracy of the information provided by information systems must be true. Usefulness of set-up information The fundamental purpose of the system is actually used, so practicality is a measure of an information system is very important landmark. This is an indicator of safety and reliability of the information system builders, the user is equally important. Information from the user's point of view should be considered as much information. Service level here mainly refers to the degree of management and decision-makers at all levels of service. inevitably have an impact from the construction of the index information systems to external influence any considerations of an information system for the external indicators considered There are 6: Sharing information sharing between systems is an important indicator of our country in this regard seriously enough, a lot of repetition to build the building of an information system to guide response system is not built to produce demonstration guiding role. Whether it is the construction of experience, development methodology and specific technical solutions, the market should be put up for everyone to learn and use. The importance of means in terms of the external environment refers to the social benefits of the system and the external society economic benefits. The amount of information is the amount of information provided to external Service level refers to the attitude and extent of the system of social services after the merger index above 24, with 20 index composed of management information systems evaluation system.

The method of determining the weights usually are: Delphi method and comparison method, AHP, entropy method according to the second $\mathrm{Y}$ and the future relationship between the composite indicators to develop a management information system to assess the quality of the system.

Correlation analysis of multi - index comprehensive evaluation method was evaluated based on experience with the ratio of science and accuracy greatly improved but not perfect First, more accurately determine the weight of the index weight is very difficult, most of the conventional method of determining. by evaluators with a personal experience, subjective, arbitrary large; secondly, the computational complexity of solving tedious, as each set of data must determine the weight and the correlation coefficient was calculated according to items one by a considerable amount.

Appears neural network system provides a new way to evaluate the problem. Neural network has many good qualities, such as adaptive, decision-making from the approximate, uncertain, even conflicting knowledge of the environment and other self-organization, and good, you can avoid artificially weighted to take into account and calculates the correlation coefficient and other sectors. Therefore, the comprehensive evaluation method based on neural network management information system is comprehensive evaluation.

Artificial neural networks are a lot of simple information unit called neuron complex network consisting of a wide range of connection used to simulate the human brain structure and behavior of the network. There are many types of neural network, BP network which is one of the most widely used Network .BP network usually consists of an input layer, a number of hidden layer and output layer, with full interconnection between layers, no interconnection between the same layer unit. has proven three-layer BP network can function with arbitrary precision approach Therefore, this article uses three layers BP network [3].

BP network learning process by forward propagation and reverse propagation of two parts. In the forward propagation process, the input samples from the input layer through the hidden layer processed and transmitted to the output layer of neurons in each layer only affects the next state layer neuron state if it is not desired output in the output layer, then transferred back propagation. in this case, the error signal from the output layer to the input layer and spread along the way to adjust 
the connection weights between the layers and layers of neurons offset value so that the error signal continues to decrease after repeated iterations, when the error is less than the allowable value, that is, network mapping requirements adapt, the training process is completed. BP algorithm there are a variety of forms, paper, a process known as the basic form of successive correction method, that each time you enter a learning sample and adjust the weights.

\section{Issues Analysis}

Select more typical 12 groups of sample data (12 group table before) as the training signal, train the network, and the remaining four groups as the test sample, mock objects to be evaluated. In the actual calculation, using Borland C4.0 programming in 586 running on the PC, a given learning accuracy of $\mathrm{E}=0.0001$, the network hidden layer neurons elected seven, the number of training $\mathrm{N}$ $=1600$, weight adjustment parameters $A=0.5$, the offset value adjustment parameter $B=0.8$, Study results are shown in Table 2, which is very close to the desired output; untrained four test samples simulation results of the evaluation and management information systems expert evaluation results fully consistent simulation results with test samples and expert evaluation of the management information system collation.

In this example, since fewer training samples, each index can be randomly generated some cases the evaluation value, and then use correlation analysis - methods for comprehensive evaluation comprehensive evaluation value extraction part of the case as a training sample, and the rest As a test sample, the network is trained and tested. In this paper, the network was trained with 28 sets of data thus generated, and the data is the same method used to produce the 12 group tested, the results showed that all the samples to assess not only the right, but untrained The test samples are all correct assessment [4].

Based on the evaluation system to establish a management information system based on the proposed evaluation method of management information system based on neural network and simulation experiment, and achieved satisfactory results the method by neural network self-learning, adaptive ability and strong fault tolerance, establish comprehensive evaluation model closer to human thinking qualitative and quantitative. trained neural network system is to evaluate the thinking of experts to connect giving way to the right on the network, so that the network can not only simulate experts on management information system for quantitative evaluation, the evaluation process and avoid human error; In addition, the model's weight is learning to get by way of example, which avoids artificial to take into account the weight and influence of subjective and uncertainty correlation coefficient. Over the years, China's college English teaching has always. Neural networks back propagation algorithm; management information systems (MIS); integrated evaluation [5].

\section{Conclusion}

Evaluation of management information systems has always been a difficult task. There are many factors which impact the information systems is good or bad, success and failure, in which there are art factors, qualitative factors, concept factors, quantitative factors and technology factors and they are crossed together, then evaluate an information system has become a very complex issue.

\section{References}

[1] Chen Jian: China University of Science and Technology, Vol. 6 (2004) No 53, p.48-51

[2] Shen Shi Yi: Neural Network Theory and Its Application, Vol. 12 (2005) No 27, p.74-76

[3] Guo Dongjiang: Management Information System, Vol. 1 (2006) No 17, p.58-63

[4] Jiao Licheng: Electronic Science and Technology, Vol. 3 (2007) No33, p.121-124

[5] Zhushuang Dong, Zhao Jiangang: Technical and Economic, Vol. 15 (2007) No42, p.12-15 